\title{
NYHA Class II or III Heart Failure: Who Will Need an Implantable Cardioverter Defibrillator (ICD)?
}

\author{
Irtiza Hasan"1,2*, Muhammad Tofazzal Hossain ${ }^{3}$, Md. Harun Ur Rashid Bhuiyan ${ }^{4}$ \\ ${ }^{1}$ Cardiology, Canterbury Christ Church University, Canterbury, UK \\ ${ }^{2}$ Internal Medicine, The University of Edinburgh, Edinburgh, UK \\ ${ }^{3}$ Cardiology, Universal Medical College, Dhaka, Bangladesh \\ ${ }^{4}$ Clinical \& Interventional Cardiology, Z.H. Sikder Cardiac Care \& Research Centre, Dhaka, Bangladesh \\ Email: *s1459527@sms.ed.ac.uk
}

How to cite this paper: Hasan, I., Hossain, M.T. and Bhuiyan, M.H.U.R. (2016) NYHA Class II or III Heart Failure: Who Will Need an Implantable Cardioverter Defibrillator (ICD)? World Journal of Cardiovascular Diseases, 6, 372-381.

http://dx.doi.org/10.4236/wjcd.2016.610042

Received: September 5, 2016

Accepted: October 15, 2016

Published: October 18, 2016

Copyright $\odot 2016$ by authors and Scientific Research Publishing Inc. This work is licensed under the Creative

Commons Attribution International

License (CC BY 4.0).

http://creativecommons.org/licenses/by/4.0/

(c) (i) Open Access

\begin{abstract}
Sudden cardiac death (SCD) is one of the most debilitating and life-threatening complications of heart failure (HF) which has challenged medical care for long. Current guidelines suggest the use of Implantable Cardioverter Defibrillator (ICD) in primary prevention of SCD in both New York Heart Association (NYHA) class II and class III heart failure. This paper critically evaluated the evidence underlying the guideline recommendation. In contrast to recent guidelines, the majority of the intervention trials conducted on the topic till date found a promising role of ICD only in the prevention of SCD in NYHA class II HF. One of the trials which found a significant role of ICD in type III heart failure was underpowered. Thus, further trials are needed to validate the use of ICD in the prevention of SCD in type III HF.
\end{abstract}

\section{Keywords}

Heart Failure, Sudden Cardiac Death, Implantable Cardioverter Defibrillator, ICD, NYHA, ESC

\section{Introduction}

Clinically, heart failure (HF) is defined as a syndrome characterized by some cardinal features (breathlessness, peripheral edema, etc.) due to a functional or structural abnormality of the heart, established by echocardiographic evidence, particularly as a reduction in ejection fraction (LVEF) [1]. The European Society of Cardiology (ESC) included an additional criterion in the definition and that is, the response to treatment 
directed towards heart failure in case of doubtful diagnoses [2]. HF is generally considered as a chronic condition traditionally resulting from left ventricular dysfunction with reduced LVEF $(\leq 40 \%)$, which can be distinguished from a small proportion of $\mathrm{HF}$ with preserved EF (HF PEF) [3]. HF is a major public health issue with substantial mortality and morbidity which also poses a challenge to clinical diagnosis and generally affects the older segment of the population [4] [5]. Various clinical conditions including ischemic and non-ischemic heart diseases, metabolic and endocrine diseases, infectious and chronic diseases, use of cardiac and non-cardiac cardiotoxic drugs are some of the common factors causing HF [6]-[11]. The prevalence of HF has an increasing trend with 23 million people worldwide being affected in 2011 alone [4]. Framingham study indicated doubling of the incidence of HF with each increasing decade of age [12]. Various population-based studies including the Framingham study, RochesterEpidemiology project and studies done by Cowie et al., Remes et al. found that the incidence rate of HF is around 1-4/1000 among the whole population and up to 16/1000 among those $>65$ years of age [1] [8]. Men are more frequently affected compared to the females [2]. The lifetime risk of developing HF is calculated to be 1 in 5 [4]. HF is also associated with high rates of hospitalization, readmissions, outpatient visits and health care cost [4]. In the early 90s, HF was the single leading cause of hospitalization and accounts for $0.2 \%$ of the admissions per annum in the European countries and has reached an epidemic proportion in many countries globally [1] [8] [12]. However, in the recent era with the development of newer and effective treatment strategies, there has been a relative reduction in hospitalization rate due to HF by $30 \%$ to $50 \%$ along with a slight decrease in mortality [1]. It is also associated with a significant portion of healthcare cost globally and exceeds $\$ 39$ billion/year in the US only [4]. The increasing prevalence of HF coupled with its substantial impact on healthcare cost and quality of life is a major public health problem globally and emphasizes the need for better and effective preventive strategies for the management of HF.

\section{New York Heart Association (NYHA) Classification of HF}

The NYHA classification is one of the most commonly used classification of HF, which is a functional classification based on patient's limitation in physical activity/exertion caused by cardiac symptoms are given in Table 1 [13].

Table 1. NYHA classification of heart failure.

\begin{tabular}{|c|c|c|}
\hline Heart failure & Symptoms & Objective Assessment \\
\hline Class II & $\begin{array}{l}\text { Slight limitation of physical activities. Comfortable at rest. Ordinary } \\
\text { physical activities may cause symptoms like fatigue, palpitation, dyspnea }\end{array}$ & $\begin{array}{l}\text { Objective evidence of minimal } \\
\text { cardiovascular disease }\end{array}$ \\
\hline Class III & $\begin{array}{l}\text { Marked limitation of physical activities. Comfortable at rest. Less than } \\
\text { ordinary activities may cause symptoms like fatigue, palpitation, dyspnea }\end{array}$ & $\begin{array}{l}\text { Objective evidence of moderately severe } \\
\text { cardiovascular disease }\end{array}$ \\
\hline Class IV & $\begin{array}{l}\text { Inability to carry out any physical activities without discomfort. Symptoms } \\
\text { of heart failure present even at rest }\end{array}$ & $\begin{array}{l}\text { Objective evidence of severe cardiovascular } \\
\text { disease }\end{array}$ \\
\hline
\end{tabular}




\section{Sudden Cardiac Death (SCD)}

HF is a chronic debilitating condition \& half of all the patients who develop HF die within 5 years of diagnosis with an annual mortality rate as high as 50\% [14] [15]. According to Rotterdam study, the age-adjusted mortality rate in HF was twice that of non-HF-related deaths [8]. Though the exact cause of death due to HF is poorly understood but according to a study done by Derfler et al. the mode of death in HF patient included metabolic (17.6\%), progressive failure (21.6\%), SCD (18.9\%), unwitnessed (9.5\%) and others (18.9\%) [16]. Another study found a fivefold increased risk of SCD in HF patients [8] [15]. SCD can be defined as an unexpected natural death occurring within a short period of time, generally $\leq 1$ hour from the onset of symptoms, usually due to cardiac causes in a person without prior fatal condition [17]. Degeneration of monomorphic ventricular tachycardia (VT) into ventricular fibrillation (VF) accounts for the majority of causes of SCD [18]. To complicate it all, VT has been documented in $85 \%$ of patients with HF especially those with LVEF $\leq 30 \%$ to $35 \%$ [18] [19]. Thus, there is a large segment of HF patients who are at risk of SCD resulting from ventricular arrhythmia which necessitates the need to focus on prevention of SCD in HF patients. The initiation of SCD in HF patients is a complex interplay and delicate balance of various underlying phenomenon including anatomic and functional cardiac substrates, transient initiating events (electrolyte, drug, stress, etc.) on the various cellular electrical circuits including prolonging action potential, delayed repolarization, reentry circuits and so on [17]. Though the mortality rate of HF increases with increase in NYHA classes but surprisingly the highest proportion of SCD is among those with less severe HF (NYHA class II and III) [18]. The risk of SCD among the NYHA class II, III, and class IV is $64 \%, 59 \%$ and $33 \%$ respectively [19].

Most of the patients who suffer from SCD do not have any antecedent arrhythmia necessitating the need to focus on primary prevention of SCD. The traditional antiarrhythmic medications are not completely able to abort the SCD [20]. Studies including AVID, CIDS, CASH, etc. demonstrated the superiority of implantable cardioverter defibrillator (ICD) over antiarrhythmic medications [21]. Bardy et al. found a $23 \%$ reduced overall mortality in HF patients using ICD [22].

\section{Implantable Cardioverter Defibrillator (ICD)}

ICD is a battery-operated device, which is placed in a pouch under the skin of chest, abdomen or collar bone and has a battery unit, which generates the pulse and 1 or 2 lead(s) placed in right ventricle \&/or right atrium, which communicates between heart \& device [23].

ICD regularly monitor heart rate and rhythm and provides defibrillatory shocks or overdrive pacing in case of an abnormal or chaotic heartbeat to terminate the arrhythmia [1] [22] [24]. The role of ICD in primary prevention of SCD depends on the severity and the etiology of left ventricular (LV) dysfunction (e.g. ischemic vs. non-ischemic) [24].

In spite of the potential role of ICD in HF, it is associated with around $25 \%$ chance of 
complication over a period of 5 years [25]. Significant complications associated with ICD include procedure related complications, failure of ICD therapy, myocardial damage by shock, inappropriate shock, psychological issues and so on [25]. Thus, there is a critical balance in the risk-benefit ratio associated with the use of ICD in primary prevention of SCD in HF patients requiring better risk stratification.

\section{Current Guidelines}

ESC guideline of 2015 or ACCF/AHA guideline of 2013 voices similar recommendation as the combined practice guideline by ACC/AHA/ESC in 2006 [1] [26]-[28]. All these guidelines recommend the use of ICD in primary prevention of SCD in symptomatic HF patients of NYHA class II and III with $\mathrm{EF} \leq 35 \%$ despite $\geq 3$ months of treatment with optimal pharmacological therapy $\&$ who are expected to survive for $>1$ year with good functional status.

In this write up we are focusing critically on the evidence underlying the use of ICD in primary prevention of SCD for NYHA class II and III HF. Various randomized controlled trials that have been done in this regard are discussed based on the etiology of LV dysfunction.

\section{Available Trial Evidence}

\section{a) Ischemic Cardiomyopathy (CM)}

\section{1) Late post-myocardial infarction (MI) trials}

These are a group of trials, which enrolled patients more than 4 to 6 weeks post-MI (Table 2). These include-

- MADIT-1 trial [29] - This trial looked at the high-risk asymptomatic patient's ages 25 to 80 years, mostly males with prior MI, non-sustained VT, LVEF $<35 \%$ or inducible sustained VT even after antiarrhythmics [29]. This trial did not differentiate between class II and III HF. The primary outcome was all-cause mortality. There was no control group, no fixed antiarrhythmic, a crossover between the arms and imbalance of antiarrhythmic between the two groups. Over 27 months of follow-up, there was a decrease in mortality in ICD group with a hazard ratio (HR) of 0.46 (0.26 to 0.82 ) with most of the benefits seen in select high-risk groups with LVEF < 26\% \& prolong QRS [29].

- MADIT II trial [30] - In contrast to MADIT I, this trial was a multicenter, randomized, unblinded trial which enrolled 1232 patients, mostly males, $>21$ years of age, who had prior MI and LVEF $\leq 30 \%$ [30]. This trial included high-risk patients with advanced left ventricular dysfunction and excluded all patients meeting MADIT I criteria. During the follow-up of average 20 months, there was a decrease in all-cause mortality in ICD arm for combined NYHA classes with a HR of 0.69 (0.5 to 0.93 ). There were more ICD related hospitalization and inappropriate shocks delivered [31]. Though not powered to look at the incidence of SCD alone but one study found that the decrease in mortality seen in this trial was entirely due to the reduction in SCD (3.8\% vs. 10\%) [32]. The benefit for NYHA class II and III 
Table 2. Trials on efficacy of ICD in prevention of sudden cardiac death in NYHA II and III heart failure.

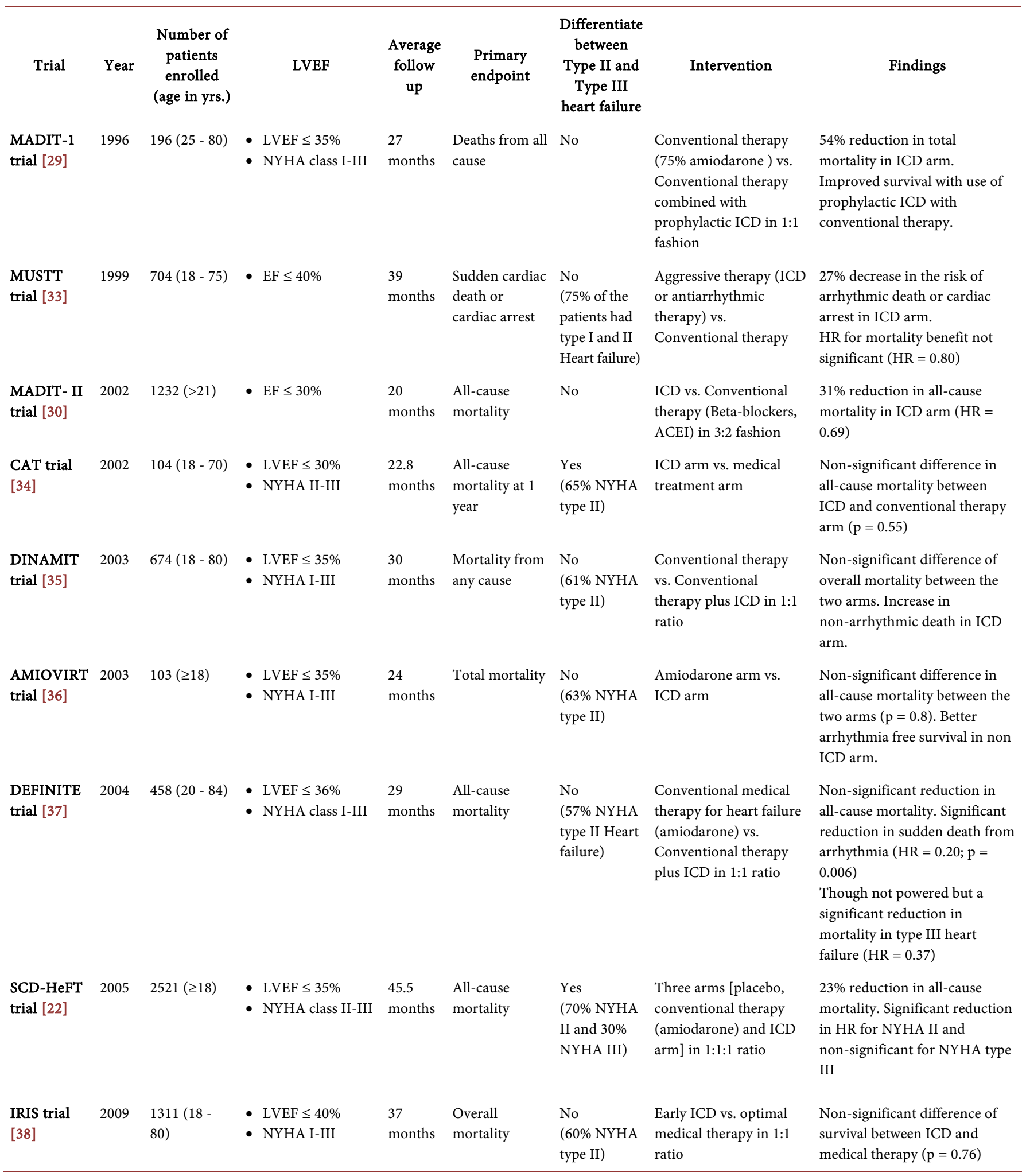

MADIT: Multicenter Automatic Defibrillator Implantation Trial; MUSTT: Multicenter Unsustained Tachycardia Trial; CAT: The Cardiomyopathy Trial; DEFINITE: Defibrillators in Non-Ischemic Cardiomyopathy Treatment Evaluation; DINAMIT: Defibrillator in Acute Myocardial Infarction Trial; SCD-HeFT: Sudden Cardiac Death in Heart Failure Trial; AMIOVIRT: Amiodarone versus Implantable Defibrillator; IRIS: Immediate Risk Stratification Improves Survival. 
were not studied individually [24].

- MUSTT trial [33] - This multicenter trial was not designed as a trial par say and was mostly looking at the management role of electrophysiological study (EPS) [33]. Patients with LVEF $\leq 40 \%$, inducible sustained VT or asymptomatic non-sustained VT was enrolled and later on randomly assigned to medical treatment or EPS guided antiarrhythmic treatment including ICD [33]. For a follow-up of 39 months, there was a reduction of SCD or arrhythmia in ICD arm with an RR of 0.24 (0.13 $0.45)$. No subgroup analyses of NYHA classes were done.

- SCD-HeFT trial [22]-This is the largest ICD trial to date involving the current symptomatic HF patients at entry with NYHA class II or III HF, reduced LVEF $\leq$ $30 \%$ and CHF within 3 months of randomization into ICD or Amiodarone or placebo arm [22]. Most of the patients were males, 70\% in NYHA class II and 30\% in NYHA class III. Over a five years follow-up, it was found that there was a $23 \%$ reduction in the primary endpoint of total mortality in ICD arm equating to $7 \%$ absolute risk reduction. The HR for NYHA class II was 0.54 (0.4 to 0.74$)$ and that for NYHA class III was 1.16 (0.84 to 1.61). There was significant mortality benefit from ICD use for NYHA class II only with no associated adverse effect seen from ICD use [22].

2) Early post-MI trials [35] [38]

- Two trials DINAMIT and IRIS evaluated the prophylactic use of ICD in the first week following MI. In both these trials, there was no difference in all-cause mortality between ICD and medical therapy but there was a high incidence of non-SCD indicating that ICD implantation in early post-MI may actually impose additional risk in HF patients.

b) Non-ischemic dilated cardiomyopathy (DCM)

HF is also common in non-ischemic DCM patients. The following trials looked at the survival benefit of ICD as a primary prevention for non-ischemic CM.

- CAT \& AMIOVIRT trials [34] [36]-Cardiomyopathy trial \& Amiodarone versus ICD trial (AMIOVERT) looked at a small number of patients with LVEF $\leq 35 \%$, where all-cause mortality was compared between ICD \& Amiodarone arm. No significant benefit was detected for ICD therapy.

- DEFINITE trial [37]-Defibrillators in Non-Ischemic Cardiomyopathy Treatment Evaluation (DEFINITE) trial evaluated the efficacy of ICD plus medical therapy versus medical therapy alone in reducing all-cause mortality in patients with reduced LVEF $\leq 35 \%$ and non-sustained VT and $70 \%$ of the patient were male. There was a non-significant decreasing trend in mortality with a significant reduction only in NYHA class III HF with HR 0.39 (0.15 - 0.90).

- SCD-HeFT [22]-As previously mentioned, it was found to be beneficial in NYHA class II with no significant benefit seen in class III HF patients.

\section{Discussion}

There have been various trials that looked at the efficacy of ICD in HF patients with different cardiomyopathies. Trials done so far on the data from various trials indicate 
that there is some definite mortality benefit with ICD implantation in $4-6$ weeks post-MI patients with ischemic CM and very low ejection fraction. The same effect was not seen for ICD implantation in early post-MI in ischemic CM patients. Most of the trials except SCD-HeFT, done on ICD implantation in non-ischemic dilated CM found no significant association between ICD implantation \& risk of SCD. Although majority of the trials did not differentiate between type II and type III HF, but they all included a significantly higher percentage ( $60 \%$ or more) of type II HF. One of the trials by Bardy et al found a significant reduction in SCD for type II HF and no effect for type III HF [22]. Thus, the mortality benefit of ICD seen in various trials is mostly validated for patients with type II HF. However, an underpowered sub-analysis done by Kadish et al found a significant mortality benefit for type III HF that emphasizes the potential for ICD in these patients [37]. As such, there is a need to critically evaluate the current evidence as well as conduct new trials to mandate the current guidelines for ICD use for the primary prevention of SCD in both NYHA class II \& III HF. Various other factors that need to be considered include-

\section{a) Evidence related to trial design and implementation:}

Almost all of the trials had a primary endpoint of reduction of all-cause mortality and hence, were possibly underpowered to look at SCD as a separate entity. More than $70 \%$ to $80 \%$ of the participants in most of the trials were males of the varied age range, which might reduce the generalizability of the results. As per ESC guideline, one of the selection criteria for ICD implantation is that the patients should be symptomatic. However, none of the other trials except the SCD-HeFT included symptomatic patients. There is a source for potential selection bias especially in MADIT I trial, where based on selection probability there is a chance of biasing the result towards ICD arm. Though the guideline indicates the use of ICD in patients with LVEF $\leq 35 \%$ but most of the trials including MADIT I, MADIT II, SCDHeFT, AVID all suggested that the most gain of benefit is for $\mathrm{EF} \leq 30$. Small sample size, frequent dropouts, non-homogeneous use of antiarrhythmics, non-uniform control arm might have reduced the power and the ability to detect significance. As most of the trials except SCD-HeFT were for 2 to 3 years, there is a lacking of trials looking at mortality benefit on prolonging use of ICD. Thus, in spite of all the evidence, there is a need for more clinical management focused research trials of longer duration, individualization of the results and case-by-case interpretation.

\section{b) Evidence related to ICD complications:}

In SCD-HeFT trial it was found that there was a five and two-fold chance of increased mortality in spite of appropriate and inappropriate shock delivered by ICD respectively [22]. This indicates the existence of competing for risk, which portrays that if SCD is reduced there is increased risk of other cause of heart failure related death. Trials have found that if the true benefit observed is similar to SCD-HeFT and persist beyond five years then the cost of primary prevention with ICD therapy is acceptable [39]. Most of the ICD effectiveness was seen with the use of single chamber devices. However, a significant proportion of ICD currently used is dual chamber [24] [29]. It 
was seen that right ventricular apical pacing in ICD recipients might actually result in ventricular asynchrony, worsening of left ventricular function \& HF. The worse outcome has been seen in patients who experienced ICD shock emphasizing the need for co-administrated optimal medical therapy. Besides, ICD has some other procedure related complications.

Thus, based on evaluation of all the trials, there are evidence for mortality benefit for primary prevention of SCD for NYHA class II but no significant benefit found for class III. So, the recommendations of ICD for primary prevention of SCD in NYHA class III requires more evidence and exact risk and benefit characterization.

\section{Conclusion}

LV dysfunction is on a rising trend worldwide and almost approaching an epidemic and exerting an enormous pressure on the healthcare system. This is challenged by the increasing prevalence of SCD in HF patients, particularly in NYHA class II \& III. Though there is a need for further research for the detection of mortality benefit for NYHA class III, ICD implantation was found to be beneficial in NYHA class II for the primary prevention of SCD. So, overall choice of ICD implantation should be based on available evidence, proper patient selection considering risk-benefit ratio, cost effectiveness and above all the patient preference.

\section{References}

[1] McMurray, J.J., et al. (2012) ESC Guidelines for the Diagnosis and Treatment of Acute and Chronic Heart Failure 2012. European Journal of Heart Failure, 14, 803-869. http://dx.doi.org/10.1093/eurjhf/hfs105

[2] Mosterd, A. and Hoes, A.W. (2007) Clinical Epidemiology of Heart Failure. Heart, 93, 1137-1146. http://dx.doi.org/10.1136/hrt.2003.025270

[3] Hogg, K., Swedberg, K. and McMurray, J. (2004) Heart Failure with Preserved Left Ventricular Systolic Function: Epidemiology, Clinical Characteristics, and Prognosis. Journal of the American College of Cardiology, 43, 317-327. http://dx.doi.org/10.1016/j.jacc.2003.07.046

[4] Bui, A.L., Horwich, T.B. and Fonarow, G.C. (2011) Epidemiology and Risk Profile of Heart Failure. Nature Reviews Cardiology, 8, 30-41. http://dx.doi.org/10.1038/nrcardio.2010.165

[5] Uddin, M., Rashid, T. and Chowdhury, S.M. (2016) Role of B-Type Natriuretic Peptide (BNP) in Heart Failure. International Journal on Disability and Human Development.

[6] Kannel, W.B. and Belanger, A.J. (1991) Epidemiology of Heart Failure. American Heart Journal, 121, 951-957. http://dx.doi.org/10.1016/0002-8703(91)90225-7

[7] McMurray, J.J. and Stewart, S. (2000) Epidemiology, Aetiology, and Prognosis of Heart Failure. Heart, 83, 596-602. http://dx.doi.org/10.1136/heart.83.5.596

[8] Mosterd, A., et al. (2001) The Prognosis of Heart Failure in the General Population. The Rotterdam Study. European Heart Journal, 22, 1318-1327. http://dx.doi.org/10.1053/euhj.2000.2533

[9] Rashid, T. and Hasan, I. (2013) Cardiotoxicity of Non Cardiovascular Drugs: A Mechanistic Point of View. BIRDEM Medical Journal, 3, 35-43.

http://dx.doi.org/10.3329/birdem.v3i1.17125 
[10] Hasan, I., Rashid, T. and Bhuiyan, M.H.U.R. (2015) PCI in Post Thrombolysis Stable STEMI Patients: A Timeline in Question. World Journal of Cardiovascular Diseases, 5, 335. http://dx.doi.org/10.4236/wjcd.2015.512039

[11] Hasan, I., Rashid, T. and Bhuiyan, M.H.U.R. (2015) Routine Primary PCI; Whether and When Necessary for the Management of NSTEMI-An Evidence Based Evaluation. World Journal of Cardiovascular Diseases, 5, 343. http://dx.doi.org/10.4236/wjcd.2015.512040

[12] Kannel, M.W.B. (2000) Incidence and Epidemiology of Heart Failure. Heart Failure Reviews, 5, 167-173. http://dx.doi.org/10.1023/A:1009884820941

[13] Bennett, J.A., et al. (2002) Validity and Reliability of the NYHA Classes for Measuring Research Outcomes in Patients with Cardiac Disease. Heart \& Lung. The Journal of Acute and Critical Care, 31, 262-270. http://dx.doi.org/10.1067/mhl.2002.124554

[14] Go, A., et al. (2013) On Behalf of the American Heart Association Statistics Committee and Stroke Statistics Subcommittee. Heart Disease and Stroke Statistics-2013 Update: A Report from the American Heart Association. Circulation, 127, e1-e240. http://dx.doi.org/10.1161/CIR.0b013e318282ab8f

[15] Tomaselli, G.F., et al. (1994) Sudden Cardiac Death in Heart Failure. The Role of Abnormal Repolarization. Circulation, 90, 2534-2539. http://dx.doi.org/10.1161/01.CIR.90.5.2534

[16] Derfler, M.C., et al. (2004) Mode of Death from Congestive Heart Failure: Implications for Clinical Management. The American Journal of Geriatric Cardiology, 13, 299-304. http://dx.doi.org/10.1111/j.1076-7460.2004.03476.x

[17] Zipes, D.P. and Wellens, H.J. (1998) Sudden Cardiac Death. Circulation, 98, 2334-2351. http://dx.doi.org/10.1161/01.CIR.98.21.2334

[18] Lane, R.E., Cowie, M.R. and Chow, A.W. (2005) Prediction and Prevention of Sudden Cardiac Death in Heart Failure. Heart, 91, 674-680. http://dx.doi.org/10.1136/hrt.2003.025254

[19] Dariusz Korczyk, T.H.M. and Kaye, G. (2012) Fast Facts: Heart Failure. Health Press Limited, Oxford, UK.

[20] Huikuri, H.V., Castellanos, A. and Myerburg, R.J. (2001) Sudden Death Due to Cardiac Arrhythmias. New England Journal of Medicine, 345, 1473-1482.

http://dx.doi.org/10.1056/NEJMra000650

[21] The AVID Investigators (1995) Antiarrhythmics versus Implantable Defibrillators (AVID) -Rationale, Design, and Methods. The American Journal of Cardiology, 75, 470-475. http://dx.doi.org/10.1016/S0002-9149(99)80583-9

[22] Bardy, G.H., et al. (2005) Amiodarone or an Implantable Cardioverter-Defibrillator for Congestive Heart Failure. The New England Journal of Medicine, 352, 225-237. http://dx.doi.org/10.1056/NEJMoa043399

[23] DiMarco, J.P. (2003) Implantable Cardioverter-Defibrillators. The New England Journal of Medicine, 349, 1836-1847. http://dx.doi.org/10.1056/NEJMra035432

[24] Myles, R.C. and Connelly, D.T. (2011) Implantable Cardioverter-Defibrillators in Heart Failure. In: McDonagh, T.A., Gardner, R.S., Clark, A.L. and Dargie, H., Eds., Oxford Textbook of Heart Failure, Oxford University Press, Oxford.

[25] Alter, P., Waldhans, S., Plachta, E., Moosdorf, R. and Grimm, W. (2005) Complications of Implantable Cardioverter Defibrillator Therapy in 440 Consecutive Patients. Pacing and Clinical Electrophysiology, 28, 926-932. http://dx.doi.org/10.1111/j.1540-8159.2005.00195.x

[26] Yancy, C.W., et al. (2013) ACCF/AHA Guideline for the Management of Heart Failure: A Report of the American College of Cardiology Foundation/American Heart Association Task Force on Practice Guidelines. Journal of the American College of Cardiology, 62, 
e147-e239. http://dx.doi.org/10.1016/j.jacc.2013.05.019

[27] Priori, S.G., et al. (2015) 2015 ESC Guidelines for the Management of Patients with Ventricular Arrhythmias and the Prevention of Sudden Cardiac Death. European Heart Journal, 36, 2793-2867. http://dx.doi.org/10.1093/eurheartj/ehv316

[28] Zipes, D.P., et al. (2006) ACC/AHA/ESC 2006 Guidelines for Management of Patients with Ventricular Arrhythmias and the Prevention of Sudden Cardiac Death-Executive Summary. European Heart Journal, 27, 2099-2140. http://dx.doi.org/10.1093/eurheartj/ehl199

[29] Moss, A.J., et al. (1996) Improved Survival with an Implanted Defibrillator in Patients with Coronary Disease at High Risk for Ventricular Arrhythmia. The New England Journal of Medicine, 335, 1933-1940. http://dx.doi.org/10.1056/NEJM199612263352601

[30] Moss, A.J., et al. (2002) Prophylactic Implantation of a Defibrillator in Patients with Myocardial Infarction and Reduced Ejection Fraction. The New England Journal of Medicine, 346, 877-883. http://dx.doi.org/10.1056/NEJMoa013474

[31] Daubert, J.P., et al. (2008) Inappropriate Implantable Cardioverter-Defibrillator Shocks in MADIT II: Frequency, Mechanisms, Predictors, and Survival Impact. Journal of the American College of Cardiology, 51, 1357-1365. http://dx.doi.org/10.1016/j.jacc.2007.09.073

[32] Greenberg, H., et al. (2004) Analysis of Mortality Events in the Multicenter Automatic Defibrillator Implantation Trial (MADIT-II). Journal of the American College of Cardiology, 43, 1459-1465. http://dx.doi.org/10.1016/j.jacc.2003.11.038

[33] Buxton, A. (1999) The Multicenter UnSustained Tachycardia Trial (MUSTT). Cardiac Electrophysiology Review, 3, 130-131. http://dx.doi.org/10.1023/A:1009981914755

[34] Bänsch, D., et al. (2002) Primary Prevention of Sudden Cardiac Death in Idiopathic Dilated Cardiomyopathy: The Cardiomyopathy Trial (CAT). Circulation, 105, 1453-1458.

http://dx.doi.org/10.1161/01.CIR.0000012350.99718.AD

[35] Dorian, P., et al. (2010) Mechanisms Underlying the Lack of Effect of Implantable Cardioverter-Defibrillator Therapy on Mortality in High-Risk Patients with Recent Myocardial Infarction: Insights from the Defibrillation in Acute Myocardial Infarction Trial (DINAMIT). Circulation, 122, 2645-2652. http://dx.doi.org/10.1161/CIRCULATIONAHA.109.924225

[36] Chinushi, M. and Aizawa, Y. (2007) Non-Pharmacological Management of Ventricular Tachycardia. Circulation Journal, 71, A97-A105.

[37] Kadish, A., et al. (2004) Prophylactic Defibrillator Implantation in Patients with Nonischemic Dilated Cardiomyopathy. The New England Journal of Medicine, 350, 2151-2158. http://dx.doi.org/10.1056/NEJMoa033088

[38] Steinbeck, G., et al. (2009) Defibrillator Implantation Early after Myocardial Infarction. The New England Journal of Medicine, 361, 1427-1436.

http://dx.doi.org/10.1056/NEJMoa0901889

[39] Mark, D.B. and Hlatky, M.A. (2002) Medical Economics and the Assessment of Value in Cardiovascular Medicine: Part I. Circulation, 106, 516-520. http://dx.doi.org/10.1161/01.CIR.0000021407.93752.7B 
Submit or recommend next manuscript to SCIRP and we will provide best service for you:

Accepting pre-submission inquiries through Email, Facebook, LinkedIn, Twitter, etc.

A wide selection of journals (inclusive of 9 subjects, more than 200 journals)

Providing 24-hour high-quality service

User-friendly online submission system

Fair and swift peer-review system

Efficient typesetting and proofreading procedure

Display of the result of downloads and visits, as well as the number of cited articles

Maximum dissemination of your research work

Submit your manuscript at: http://papersubmission.scirp.org/

Or contactwjcd@scirp.org 